Keywords: Dementia; Physicians; Practice guideline; Hong Kong.

\title{
A survey of physician practices in managing people with dementia in Hong Kong
}

\author{
Kor Pui-kin Patrick \\ Lai Kam-Yuk Claudiab,* \\ Liu Yat-Wa Justinac \\ Dai Lok-Kwan Davidd \\ Ting Shuk-Man Sylvia ${ }^{\mathrm{e}}$ \\ Kin Choif \\ a Clinical Instructor, School of Nursing, \\ The Hong Kong Polytechnic University \\ b Professor, School of Nursing, The Hong \\ Kong Polytechnic University \\ ${ }^{c}$ Assistant Professor, School of Nursing, \\ The Hong Kong Polytechnic University \\ d Consultant, Medical \& Geriatrics \\ Department, Prince of Wales Hospital, \\ Hong Kong \\ e Advanced Practice Nurse, School of \\ Nursing, The Hong Kong Polytechnic \\ University \\ ${ }^{f}$ Former President, The Hong Kong \\ Medical Association \\ CHINA
}

\begin{abstract}
Background and Objectives: Managing patients with dementia (PWD) in accordance with established clinical practice guidelines is important in providing optimal care. However, information about the actual processes of diagnosis and treatment is scanty. The objective of this paper is to explore the daily practices and management of PWD by physicians in Hong Kong.

Methods: A survey was conducted to explore the practices of local physicians in managing PWD. A questionnaire was sent to the Hong Kong Medical Association, which represents $61 \%$ of all locally registered medical practitioners.

Results: The Mini-Mental State Examination (MMSE) was the most common screening instrument (89.4\%), followed by the Clock drawing test (29.3\%). The most frequently used imaging tests were computed tomography $(67.9 \%)$. Tests for thyroid-stimulating hormones (85.9\%) and vitamin B12 (74.9\%), as well as the Venereal Disease Research Laboratory Test $(74.9 \%)$, were frequently performed to rule out reversible causes. Cholinesterase inhibitor $(69.7 \%)$ and N-Methyl-D-asparate $(44.1 \%)$ were the most commonly used anti-dementia medications. The most common reason for referring patients to specialists was the "occurrence of severe behavioral and psychiatric symptoms (BPSD)" (59.6\%).
\end{abstract}


Discussion: Most physicians in Hong Kong can select cognitive assessment tools, laboratory tests, and imaging tests recommended by several practice guidelines. Primary care physicians should be able to manage PWD who are in stable condition and without severe BPSD. Better education of physicians in the diagnosis and management of dementia is needed to ensure that all physicians practice in ways consistent with the established practice guidelines.

Received: 5 February 2015

Revised: 14 May 2015

Accepted: 19 May 2015

\section{Introduction}

Dementia is a growing public health concern, with prevalence rates ranging from $2 \%$ to $8.5 \%$ among people aged 60 and over ${ }^{1}$. About two-thirds of dementia cases may remain undetected ${ }^{2}$. Despite the importance of early diagnosis, there are multiple barriers to the processes of assessing, diagnosing, and treating dementia, leading to under-recognition of Alzheimer's disease and other forms of dementia $^{3,4}$. Factors contributing to a delay in diagnosis include the failure to recognize symptoms ${ }^{5}$, time pressures on practitioners in urban practice settings, the limited availability of diagnostic and community support services for those working in rural areas ${ }^{6}$, and difficulty in getting specialist appointments ${ }^{5}$.

Clinical practice guidelines provide physicians with recommendations on how to manage a patient with dementia (PWD). The first guidelines on dementia care were developed in 1984 in the United States ${ }^{7}$. In the past few decades, several guidelines have been developed by different national and international societies, academies, and institutes. In Hong Kong, there are no specific local guidelines for physicians. We extracted and summarized recent guidelines from the Alzheimer's Society of the United Kingdom, the European Federation of Neurological Societies (EFNS), the American Psychiatric Association, the American Academy of Neurology (AAN), and the Canadian Consensus Conference. We concluded that the dementia management processes recommended by the guidelines should include formal memory testing, imaging, laboratory testing, interventions, counseling, and specialist referrals. According to these guidelines, formal cognitive assessments should be performed using validated assessment tools such as the MMSE, the Clock drawing test, the Montreal Cognitive Assessment, DemTect, the 7-Minute Screen, the General Practitioner Assessment of Cognition, and so on ${ }^{8-11}$. Computed tomography and magnetic resonance imaging are recommended for imaging tests in $\mathrm{PWD}^{9}$. Guidelines also recommend blood tests, including a complete blood count (to rule out anemia), thyroid-stimulating hormones (to rule out hypothyroidism), serum electrolytes (to rule out hyponatremia), serum calcium (to rule out hypercalcemia), serum fasting glucose (to rule out hyperglycemia), and serum vitamin B12, serum folic acid, syphilis, and HIV screening (for individual cases at high risk or where there are suggestive clinical features) $)^{9-11}$. Pharmacological interventions including antidementia medications such as cholinesterase inhibitors and N-Methyl-D-aspartate (Memantine) are recommended, and other medications such as antipsychotics, antidepres- 
sants, anxiolytic drugs, and hypnotic drugs may be required according to the signs and symptoms of patients ${ }^{12,13}$. Counseling on stress management, financial planning, medication management, home safety risks, ongoing activity, and functional problems should be provided to patients with dementia and their families ${ }^{11,12,14}$. Referrals can be made to memory clinics or specialists including geriatricians, psychiatrists, or neurologists ${ }^{13,15}$.

Practice guidelines are of assistance to doctors in making diagnoses and prescribing treatments for complicated diseases such as dementia ${ }^{16}$. Practice guidelines also set up a standard approach to managing dementia, and the failure to proceed in accordance with clinical practice guidelines can result in adverse outcomes such as delays in reaching a diagnosis, institutionalization, and the emergence of behavioral and depressive symptoms in patients ${ }^{17}$. Syndromes including depression or mild cognitive impairment can easily be missed if the doctor does not follow the guidelines for using the assessment tools ${ }^{18,19}$. Some studies have shown that physicians may not be following the guidelines in their daily practice ${ }^{20,21}$. Their training and experience, geography and resource constraints, along with rapid changes in the guidelines, contribute to variations in the management of $\mathrm{PWD}^{22,23}$. The objective of this paper is to explore the daily practice and management of PWD by physicians in Hong Kong with regard to assessment, treatment, and attitude.

\section{Methods}

A postal survey of physicians in Hong Kong was conducted from December 2010 to March 2011. Dementia is a common problem in the elderly and the number of sufferers is increasing. Dementia should not be managed only by specialists. Any general practitioner or physician should be able to make the diagnosis of dementia and provide appropriate treatment for a PWD in stable condition and without severe BPSD. Therefore, we conducted a broad survey via the Hong Kong Medical Association, which represents 61\% of all locally registered medical practitioners. We designed a questionnaire based on a review of the literature and drawing on the expertise of clinicians in relevant specialties. Our questionnaire is comprised of four sections. Section 1 contains questions designed to collect demographic data on the respondents. Section 2 consists of 14 questions exploring how physicians diagnose and manage dementia. The design of section 3 was adapted, with approval, from the instrument developed by Turner et al. The original instrument contained 10 statements to evaluate the confidence of GPs in treating PWD, an aim that different slightly from the objectives of this survey. Therefore, two of the statements about family concerns were deleted. With the addition of four new statements, section 3 now contains a total of 12 statements to investigate the respondents' attitudes to treating PWD. Statements 1 to 3 relate to physicians' perceptions about treating PWD. Statements 4 and 5 investigate their pro-activeness in making the diagnosis and treating PWD, were added to the new scale for the present study. The respondents were asked to rate their level of acceptance of each statement on a 7-point Likert scale ranging from 1 (strongly disagree) to 7 (strongly agree). Section 4 consists of 7 questions about what training the respondents had received in managing dementia and their educational background. The content validity index of the questionnaire was 3.24 out of 4 , as assessed by a panel of experts in the field. A pilot survey was conducted on a convenience sample of 10 GPs. No particular concerns were raised during the pilot study. Ethical approval was 
obtained from the Human Subjects Ethics Sub-committee of The Hong Kong Polytechnic University. To maintain anonymity, the mailing of the questionnaire was managed by HKMA staff not involved in the survey. The research team had no access to information on HKMA members. All of the questionnaires that were received were checked for missing data. In the case of questionnaires that were improperly completed, the team immediately contacted the respondent through the HKMA secretary for clarifications and asked him or her to fill in the missing information. Four weeks after sending out the initial postal questionnaire, we checked the serial number of every questionnaire that had been received and informed the secretary of the HKMA about any missing serial numbers. The secretary then sent out a reminder and a second copy of the questionnaire to those practitioners who had failed to submit the questionnaire, as determined by the missing serial numbers.

\section{Results}

\section{Demographic data}

Four hundred and forty-eight questionnaires were received, of which 370 had been fully completed. Forty-eight of the questionnaires had been partially completed, and 12 respondents indicated that they had either retired or were no longer practicing. Of the partially completed questionnaires, four were excluded from the analysis as the respondents had not given answers to specific key questions or their answers could not be understood. The final sample was 414 respondents. In our study, "specialist" $(n=82)$ has been broadly defined as a physician who has obtained a quotable diploma in geriatric medicine or received other specialized training in dementia, with about seven hours of training in the last 12 months. Please refer to Table 1 for the details.

\section{Dementia management practices}

\section{Cognitive assessment}

The "MMSE" is the most commonly used instrument to screen for dementia $(89.4 \%)$, followed by the "Clock Drawing Test" $(29.3 \%)$ and the "Abbreviated Mental Test" (18.6\%). Six percent of the respondents did not use any screening instruments, and $3.7 \%$ used "other" instruments, including the "Rivermead Behavioral Memory Test," the "Double-headed daisy" (a test used to check any neglect of brain functions), "Clinical dementia rating and clinical interview," "Diagnosis already made in HA (Hospital Authority) hospital," and "History" - from history provided by family members (Table 2 ).

\section{Laboratory tests}

The respondents were asked about which laboratory tests they commonly used to diagnose dementia. The five most common responses were "thyroid-stimulating hormone (TSH)" (85.9\%), "vitamin B12" (74.9\%), the "Venereal Disease Research Laboratory Test (VDRL)" (74.9\%), "complete blood count (CBC)" (73.8\%), and "fasting blood glucose" $(61.8 \%)$. Ten percent of the respondents did not conduct any laboratory investigations. The respondents who selected "others" (18.8\%) mentioned: "All blood facts available $(\mathrm{a} / \mathrm{v})$ to rule out organic causes," "blood lipid," "erythrocyte sedimentation rate (ESR)," "folate (red cell)," "homocysteine cholesterol high sensitivity C reactive protein (CRP)," "ceruloplasmin (Cp)," "human immunodeficiency virus (HIV) (if young)," "non-contrast CT brain/MRI brain scan," "chest x-ray (CXR)," 


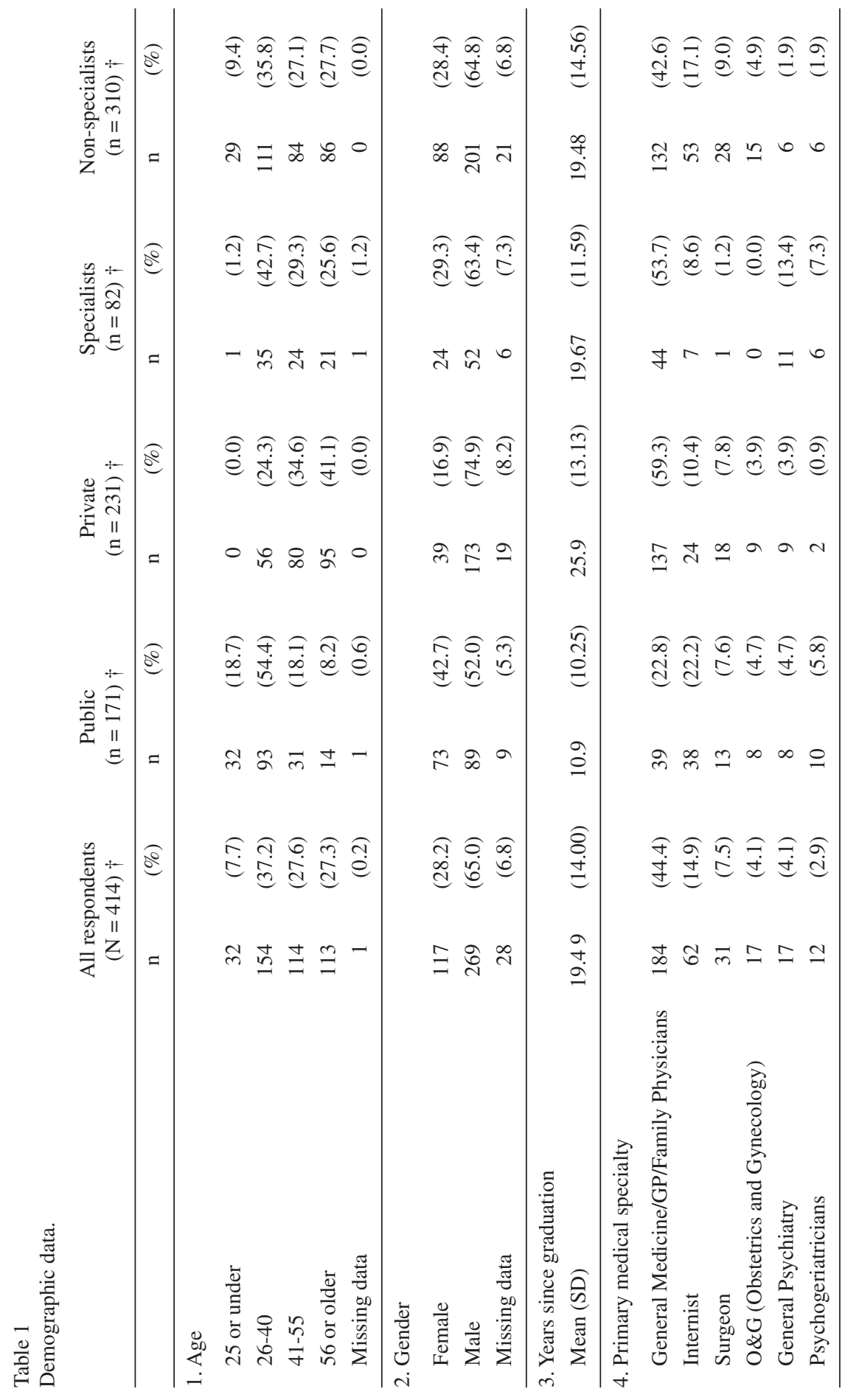









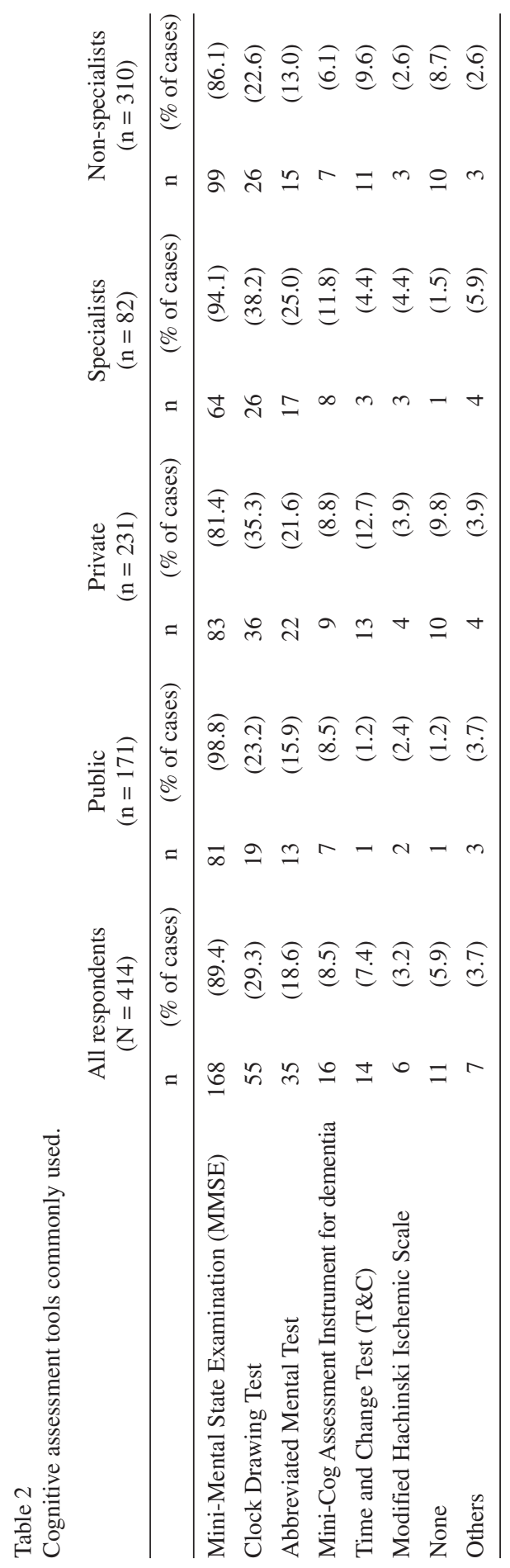

"electroencephalogram (EEG)," "test for heavy metals \& rare toxic chemicals (e.g., copper)," and "all tests are expensive, therefore refer to government" (Table 3).

\section{Imaging tests}

We asked the respondents about the imaging test(s) that they used most frequently for diagnosing dementia. The three most common responses were "computed tomography (CT)" (67.9\%), "magnetic resonance imaging (MRI)" (30.6\%), and "magnetic resonance spectroscopy" (2\%). Fourteen percent of the respondents did not order any imaging tests (Table 3).

\section{Anti-dementia medications}

Regarding the types of anti-dementia medications respondents may prescribe to their PWD, the drug most commonly used was a "cholinesterase inhibitor, e.g. Donepezil, Rivastigmine or Galantamine" (69.7\%). "NMethyl-D-aspartate (NMDA) receptor antagonist, e.g. Memantine" was used by $44.1 \%$ of the respondents, whereas $22.6 \%$ used "Nonsteroidal anti-inflammatory drugs (NSAID) such as Aspirin." Thirteen per cent of the respondents did not prescribe any anti-dementia medications to their patients (Table 4).

\section{Oral supplements}

Over $40 \%$ of the respondents did not prescribe any supplements to PWD. The supplements included "'vitamin E" (32.6\%), "Piracetam (Nootropil)" (26.3\%), "Gingko biloba" $(23.7 \%)$, and "Selegiline" (7.9\%). Respondents who selected "other" supplements (7.4\%) mentioned "Bco, multi-vitamins, B6, B12, folic acid," "Duxaril," "hydergine," "Phosphatidylserine, Dehydroepiandrosterone (DHEA), omega 3, fatty acids," "Sermion (i.e., Nicergoline)," and "Consult geriatrician/psychiatrist for prescription" (Table 4). 


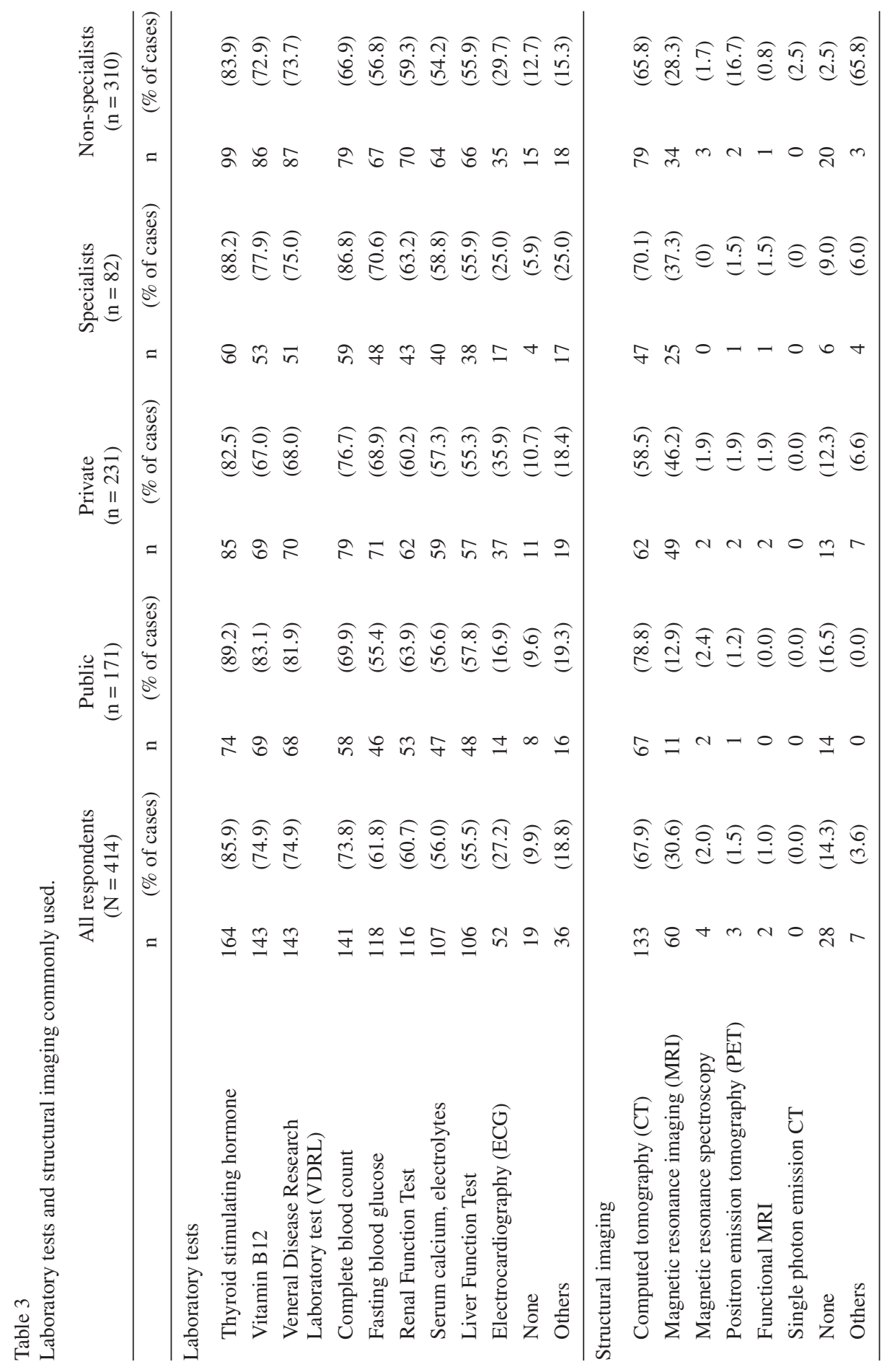




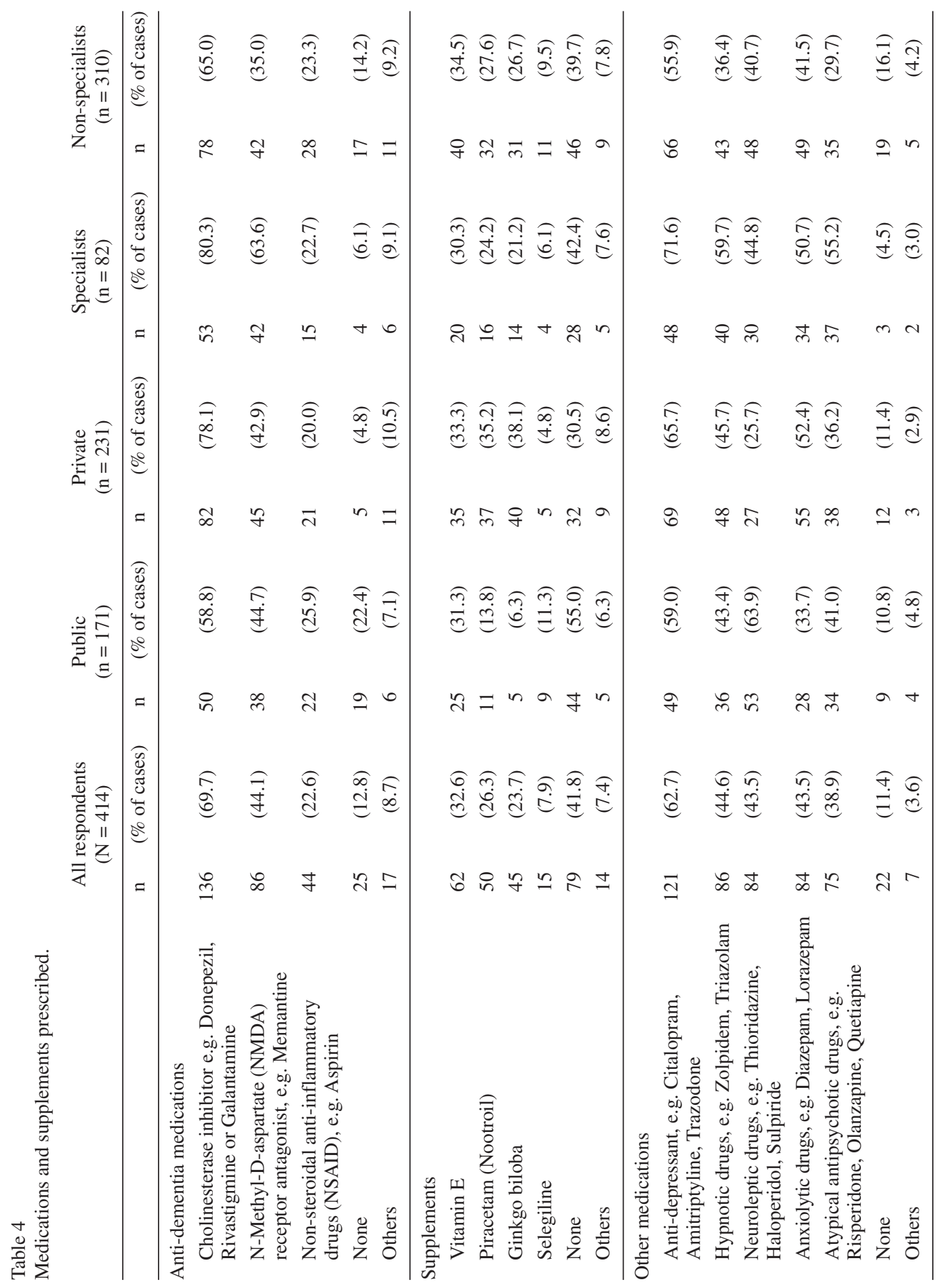




\section{Non anti-dementia medications}

From responses to the question on other medications prescribed to PWD, it seems that in addition to anti-dementia medications, other types of medication were also commonly prescribed. Psychiatric medications were common, including, for example, "anti-depressants, such as Citalopram, Amitriptyline, Trazodone" (62.7\%), "Hypnotic drugs, such as Zolpidem, Triazolam" (44.6\%), "neuroleptic drugs, such as Thioridazine, Haloperidol, Sulpiride" (43.5\%), "anxiolytic drugs, such as Diazepam, Lorazepam" (43.5\%), and "atypical antipsychotic drugs, such as Risperidone, Olanzapine, Quetiapine" (38.9\%). Eleven percent of the respondents did not prescribe any other medications (Table 4).

\section{Reasons for referring specialists}

When asked under what circumstances the respondents would consider referring their patients to specialists such as geriatricians, psychogeriatricians, or neurologists, their responses were: "Occurrence of severe BPSD" (59.6\%), "For treatment and monitoring" (56.5\%), "Request by patients/ family members" $(54.5 \%)$, "For specific treatment required by patients" (54.0\%), "For diagnosis" $(42.7 \%)$, and "Occurrence of severe medication side effects" (41.7\%). For those who selected "Others" (4.3\%), their reasons included, "Assessment of mental fitness for consent," "Fitness of OT consent," "For court case," "Complex presentation," "Organic causes, such as neurosurgical conditions," "Suspected secondary causes of dementia," or "Young demented patients" (Table 5).

\section{Role of doctor in the management of $P W D$}

When the respondents were asked about the role of the medical doctor in managing patients with dementia, most chose "Diagnosis" (80.0\%), "Screening" (78.1\%), "Referring patients to specialists" (75.9\%), and
"Educating family members" (75.4\%). Other roles cited included "Referring patients to other community support" $(63.0 \%)$, "Treatment" (62.0\%), "Monitoring the effectiveness of the treatment" (56.0\%), and "Management of patients' behavioral and psychiatric symptoms (BPSD) preferably with input from geriatricians/psychogeriaticians for difficult cases" $(50.1 \%)$ (Table 6).

\section{Discussion}

Although there are guidelines for physicians on how to manage PWD, there are wide variations in their daily practice with regard to assessment tools, reasons for referrals, and treatment ${ }^{21,22,23}$. To make accurate diagnoses, medical doctors use a wide range of screening instruments that are validated and recommended in the guidelines, such as MMSE. A systematic review found a broad variation in the use of formal memory testing during assessments. Some physicians asked questions during history taking to assess the memory of a PWD instead of using screening instruments $^{22}$. Like other surveys ${ }^{24,25}$, our results show that MMSE is the most commonly used tool $(89.4 \%)$ in cognitive assessments. However, it was surprising that $5.9 \%$ of the respondents in our survey did not use any screening instruments. The clinical guidelines of the EFNS state that the use a screening tool in cognitive assessments is essential to diagnosing dementia and should be performed on all patients ${ }^{11}$. Although the MMSE is commonly used, some argue that it may not be practical for use in primary care, as it takes about 20 minutes to complete ${ }^{23}$. As a result, different types of validated screening tools for cognitive assessments have been in the last few decades. The guidelines of the Alzheimer's Society of the UK, based on ex- 

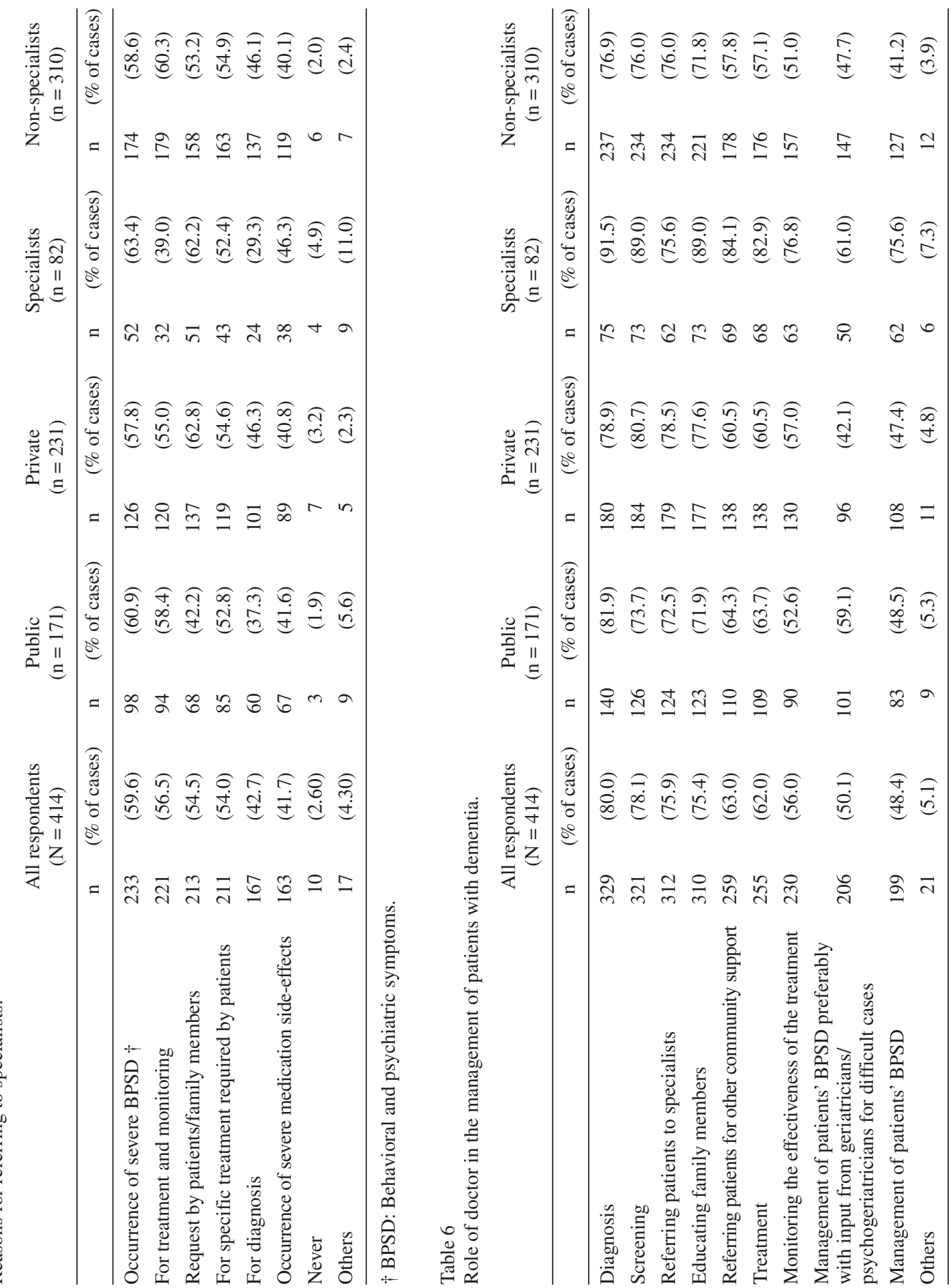

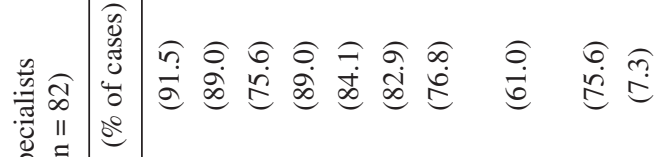
क्ष

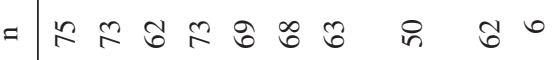

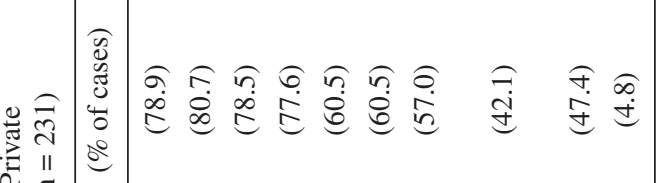
है

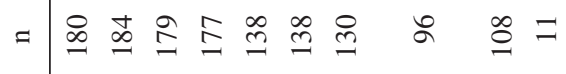

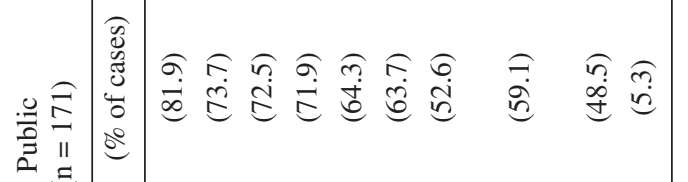



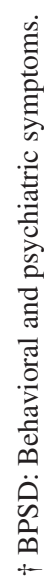

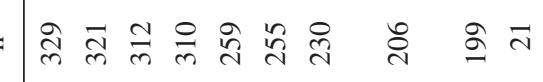

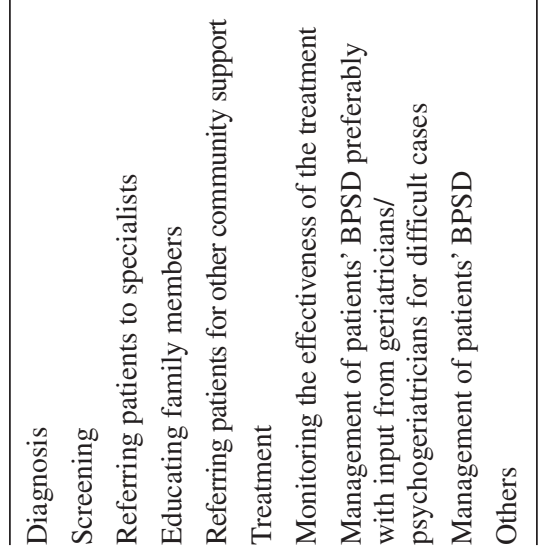


pert panel advice and taking feasibility into consideration, suggest the use of different assessment tools for primary and specialized clinical settings ${ }^{8}$. The Alzheimer's Association of the US also mentions that there is no single test that covers every situation; guidelines only inform physicians about the different assessment tools that are available, and how they may be used in clinical practice $^{26}$. According to our finding, the commonly used assessment tools in Hong Kong, including AMTS and MMSE, are recommended under different guidelines. The MMSE is the most common assessment tool used in Hong Kong for cognitive screening. Choosing the appropriate assessment tools is the first step. It is more important to interpret the results correctly. It is encouraging to see that more than $90 \%$ of physicians in Hong Kong, including both specialists and nonspecialists, selected the assessment tools recommended by the practice guidelines, but it is also important to find out why $5.9 \%$ of the respondents did not follow the guidelines to use any screening instruments.

Structural imaging should be used to evaluate every PWD with a Level A recommendation in the practice guidelines, as CT and MRI can help to exclude secondary causes of dementia, such as tumors or inflammatory disease, and also to identify patients with comorbid cerebrovascular disease (mixed dementia) in some countries ${ }^{11,27}$. Most respondents $(97.9 \%)$ prescribed imaging tests in their daily practice. Our results also showed that nearly half of the respondents in the private sector used CT (58.8\%) and MRI (46.2\%). Most physicians in public hospitals preferred using CT $(78.8 \%)$ to MRI (12.9\%). It is interesting to examine why only a few respondents in the public sector use MRI, but it is more important to explore the differences in sensitivity between CT and MRI in dementia screening. A systematic review found that there is insufficient evidence to suggest that MRI is superior to CT in screening cerebrovascular changes in autopsy-confirmed and clinical cohorts of vascular dementia, Alzheimer's disease, and "mixed dementia" 28 . Therefore, both CT and MRI can be used in the initial screening.

Apart from imaging tests, the most commonly used laboratory investigations in our survey were blood tests for TSH, vitamin B12, and VDRL (74.9-85.9\%). A recent survey reviewed the potentially reversible conditions in dementia and mild cognitive impairment in a geriatric clinic and found that the causes were mainly hypothyroidism and vitamin B12 deficiency ${ }^{29}$. Therefore, guidelines from the EFNS and AAN support routine blood tests for TSH and vitamin B12 11,30. In our result, more than half of the respondents $(74.9 \%)$ performed a VDRL test. Although syphilis is one of the causes of cognitive impairment, the practice guidelines suggest that the VDRL test should be only used on high-risk patients and those with evidence of dementia in their clinical presentation $^{11}$. Ten percent of the respondents did not follow the guidelines to perform any routine blood test for PWD, so some of the reversible causes of cognitive impairment may have been missed.

Cholinesterase inhibitors and N-Methyl-Daspartate are medications suggested under different guidelines for PWD. A similar local survey, conducted in 2008, noted a trend of decrease in the prescription of cholinesterase inhibitors for the management of $\mathrm{PWDs}^{31}$. From the results in the study, this trend seems to be changing. In our survey, cholinesterase inhibitors were the most common medication (70\%) used for managing PWD. In their guidelines, the American Academy of Family Physicians and the American College of Physicians state that cholinesterase inhibitors can be used to treat mild to moderate dementia, but 
that the improvements in dementia that have been achieved with these pharmacologic agents have only been demonstrated in tests but not in daily life ${ }^{30}$. The guidelines suggest that cholinesterase inhibitors should only be prescribed for PWD after an individual assessment that takes into account the benefits and risks of taking these medications. In our study, we cannot assess the appropriate use of pharmacological interventions prescribed by doctors, but the results suggest that the commonly used anti-dementia medications for PWD in Hong Kong are similar to those mentioned in the practice guidelines.

Besides anti-dementia medications, medical doctors prescribe other medications and supplements according to a patient's symptoms. Guidelines suggest using different types of antipsychotics, antidepressants, and anxiolytic and hypnotic drugs after a comprehensive assessment of the PWD. In our sample, $26.3 \%$ of the respondents prescribed Piracetam. However, a Cochrane review in 2008 showed inconsistent results on the use of Piracetam in treating PWD. It is surprising to note that Piracetam is still being prescribed in Hong Kong. A focus group study by Pimlott et al. (2009) revealed that family physicians were only minimally aware of the contents and details of clinical practice guidelines ${ }^{32}$. A similar survey found that most physicians relied mainly on their own clinical experience to prescribe treatments for $\mathrm{PWD}^{33}$, rather than on the current guidelines. In the guidelines that we reviewed, Piracetam is not mentioned or recommended for use in treating PWD.

Medical physicians play a wide variety of roles in the management of PWD. Several studies have already discussed the roles played by primary physicians in caring for PWD and queried their ability to make appropriate diagnoses ${ }^{3,21,34}$. There is no doubt that signs of early dementia are easy to miss or difficult to identify ${ }^{35}$. Most respondents (more than $75 \%$ ) in our survey identified their roles as making diagnoses, screening, and referring patients to specialists. Only $62 \%$ of respondents regarded their role as providing treatment for patients with dementia. The results suggest that physicians in Hong Kong prefer to refer patients to specialists rather than to provide treatment for PWD. According to a review by Chow et al. (2009), family practitioners today should be able to make the diagnosis of dementia and provide appropriate treatment for PWD who are in stable condition and without severe $\mathrm{BPSD}^{36}$. It is interesting that only about half of the respondents considered it their role to provide treatment for PWD. Several studies have emphasized the importance of medical physicians in making both appropriate diagnoses and referrals, as they are at the front line in detecting dementia $^{21,37}$. The most common reason for making referrals was the "occurrence of BPSD" (59.6\%), followed by "treatment and monitoring" (56.5\%). Several practice guidelines indicate that it is the responsibility of the medical doctor to make referrals, but do not mention the specific circumstances ${ }^{4,11,38,39}$. Inappropriate referrals may increase the demand and workload of specialists. Further indepth exploration and studies should be conducted so that recommendations can be made on the specific circumstances in which it is appropriate to make a referral.

\section{Limitations}

The low response rate limits the generalizability of the findings to all medical physicians in Hong Kong. However, in terms of response rate our survey ranked the second highest among surveys undertaken by the HKMA. Also, those who chose to respond might have had some interest in or commit- 
ment to dementia and dementia care. There was a self-selection bias in participants, which commonly seen in surveys. In addition, although we categorized the respondents into specialists and non-specialists, we did not explore the practices and outcomes of different sub-specialties of practitioners. In the future, researchers would examine the practice patterns of those in various specialties.

\section{Conclusion}

Most of the respondents, whether specialists and non-specialists, were able to select the cognitive assessment tools and laboratory and imaging tests recommended by the practice guidelines. Medical physicians should not only make referrals to specialists, but also diagnose and manage PWD who are in stable condition and do not have severe BPSD. A few medical physicians may not prescribe medications in accordance with the practice guidelines. It is important to find a way to link the practice guidelines and current practices. Better education of physicians in managing PWD is needed to help reduce the demand on specialists and the healthcare system. The findings obtained in this survey are highly informative with regard to the practices of local physicians in dementia care, and provide useful information for professional development.

\section{References}

1. Prince M, Bryce R, Albanese E, Wimo A, Ribeiro W, Ferri CP. The global prevalence of dementia: A systematic review and metaanalysis. Alzheimers Dement. 2013; 9(1): 63-75.
2. Wilkins CH, Wilkins KL, Meisel M, Depke M, Williams J, Edwards DF. Dementia undiagnosed in poor older adults with functional impairment. J Am Geriatr Soc 2007; 55(11): 1771-6.

3. Olafsdóttir M, Foldevi M., Marcusson J. Dementia in primary care: Why the low detection rate? Scand J Prim Health Care. 2001; 19(3): 194-8.

4. Iliffe S, Robinson L, Brayne C, Goodman C, Rait G, Manthorpe J, et al. DeNDRoN Primary Care Clinical Studies Group. Primary care and dementia: 1. diagnosis, screening and disclosure. Int J Geriatr Psychiatry. 2009; 24(9): 895-901.

5. Teel CS. Rural practitioners' experiences in dementia diagnosis and treatment. Aging Ment Health. 2004; 8(5): 422-9.

6. Boise L, Camicioli R, Morgan DL, Rose JH, Congleton L. Diagnosing Dementia: Perspectives of primary care physicians. Gerontologist. 1999; 39(4): 457-64.

7. McKhann G, Drachman D, Folstein M, Katzman R, Price D, Stadlan EM. Clinical diagnosis of Alzheimer's disease: Report of the NINCDS-ADRDA Work Group under the auspices of the Department of Health and Human Services Task Force on Alzheimer's disease. Neurology. 1984; 34(7): 939-44.

8. Ballard C, Burns A, Corbett A, Livingston G, Rasmussen J. Helping you to assess cognition: A practical toolkit for clinicians. England: Alzheimer s Society, 2013.

9. Feldman HH, Jacova C, Robillard A, Garcia A, Chow T, Borrie M, et al. Diagnosis and treatment of dementia: 2. Diagnosis.(Disease/Disorder overview). CMAJ. 2008; 178(7): 825-36.

10. Petersen RC, Stevens JC, Ganguli M, Tangalos EG, Cummings JL, DeKosky ST. Practice parameter: Early detection of dementia: Mild cognitive impairment (an evidence- based review) Report of the Quality Standards Subcommittee of the American Academy of Neurology. Neurology. 2001; 56(9): 1133-42.

11. Sorbi S, Hort J, Erkinjuntti T, Gainotti G, Gurvit H, Nacmias B. et al. EFNS Scientist Panel on Dementia and Cognitive Neurology. EFNS- ENS Guidelines on the diagnosis and management of disorders associated with dementia. Eur J Neurol. 2012; 19(9): 1159-79.

12. Herrmann N, Gauthier S. Diagnosis and treatment of dementia: 6. Management of severe Alzheimer disease (Clinical report). CMAJ. 2008; 179(12): 1279-87.

13. Rabins PV, Blacker D, Rovner BW, Rummans T, Schneider LS, Tariot PN. et al. American Psychiatric Association practice guideline for the treatment of patients with 
Alzheimer's disease and other dementias. Second edition. Am J Psychiatry. 2007; 164(12): 5-56.

14. Hogan DB, Bailey P, Black S, Carswell A, Chertkow H, Clarke B. et al. Diagnosis and treatment of dementia: 4. Approach to management of mild to moderate dementia (Clinical report). CMAJ. 2008; 179(8): 787-93.

15. Patterson C, Feightner JW, Garcia A, Hsiung G-R, MacKnight C, Sadovnick AD. Diagnosis and treatment of dementia: 1. Risk assessment and primary prevention of Alzheimer disease. CMAJ. 2008; 178(5): 548-56.

16. De Lepeleire J, Wind AW, Iliffe S, Moniz-Cook ED, Wilcock J, Gonzalez VM, et al. The primary care diagnosis of dementia in Europe: An analysis using multidisciplinary, multinational expert groups. Aging Ment Health. 2008; 12(5): 568-76.

17. Chodosh J, Mittman BS, Connor KI, Vassar SD, Lee ML, DeMonte RW, et al. Caring for patients with dementia: How good is the quality of care? Results from three health systems. J Am Geriatr Soc. 2007; 55(8): 1260-8.

18. Brown EL, Raue P, Halpert KD, Adams S, Titler MG. Detection of depression in older adults with dementia. J Gerontol Nurs. 2009; 35(2): 11-15. Review.

19. Stephan BCM, Brayne C, Mckeith IG, Bond J, Matthews FE. Medical Research Council Cognitive Function and Ageing Study. Mild cognitive impairment in the older population: Who is missed and does it matter? Int $\mathbf{J}$ Geriatr Psychiatry. 2008; 23(8): 863-71.

20. Rosen CS, Chow HC, Greenbaum MA, Finney JF, Moos RH, Sheikh JI, et al. How well are clinicians following dementia practice guidelines? Alz Dis Assoc Dis. 2002; 16(1): 15-23.

21. Wilcock J, Iliffe S, Turner S, Bryans M, O'Carroll R, Keady J, et al. Concordance with clinical practice guidelines for dementia in general practice. Aging Ment Health. 2009; 13(2): 155-61.

22. Sivananthan SN, Puyat JH, Mcgrail KM. Variations in self reported practice of physicians providing clinical care to individuals with dementia: A systematic review. J Am Geriatr Soc. 2013; 61(8): 1277-85.

23. Fortinsky RH, Zlateva I, Delaney C, Kleppinger A. Primary care physicians' dementia care practices: Evidence of geographic variation. Gerontologist. 2010; 50(2): 179-91.

24. Thyrian JR, Hoffmann W. Dementia care and general physicians - A survey on prevalence, means, attitudes and recommendations. Cent Eur J Public Health. 2012; 20(4): 270-5.
25. Belmin J, Min L, Roth C, Reuben D, Wenger N. Assessment and management of patients with cognitive impairment and dementia in primary care. J Nutr Health Aging 2012; 16(5): 462-7.

26. Cordell CB, Borson S, Boustani M, Chodosh J, Reuben $\mathrm{D}$, Verghese $\mathrm{J}$, et al. Alzheimer's Association recommendations for operationalizing the detection of cognitive impairment during the Medicare Annual Wellness Visit in a primary care setting. Alzheimers Dement. 2013; 9(2): 141-50.

27. Huffman GB. The Role of CT and MRI in the Evaluation of Dementia. Am Fam Physician. 2001; 63(7): 1417-8.

28. Beynon R, Sterne JA, Wilcock G, Likeman M, Harbord RM, Astin M, et al. Is MRI better than CT for detecting a vascular component to dementia? A systematic review and meta-analysis. BMC Neurol. 2012; 12(1): 33.

29. Muangpaisan W, Petcharat C, Srinonprasert V. Prevalence of potentially reversible conditions in dementia and mild cognitive impairment in a geriatric clinic. Geriatr Gerontol Int. 2012; 12(1): 59-64.

30. Graham L. AAFP and ACP release guideline on dementia treatment. Am Fam Physician. 2008; 77(8):1173-5.

31. Maeck L, Haak S, Knoblauch A, Stoppe G. Primary care physicians' attitudes related to cognition enhancers in early dementia: a representative eight- year follow- up study in Lower Saxony, Germany. Int J Geriatr Psychiatry. 2008; 23(4): 415-21.

32. Pimlott NJG, Persaud M, Drummond N, Cohen CA, Silvius JL, Seigel K, et al. Family physicians and dementia in Canada: Part 1. Clinical practice guidelines: awareness, attitudes, and opinions. Can Fam Physician 2009; 55(5): 506-7.

33. Thyrian JR, Hoffmann W. Dementia care and general physicians - a survey on prevalence, means, attitudes and recommendations. Cent Eur J Public Health. 2012; 20(4): 270-5.

34. Cahill S, Clark M, Walsh C, O'Connell H, Lawlor B. Dementia in primary care: The first survey of Irish general practitioners. Int J Geriatr Psychiatry. 2006; 21(4): 319-24.

35. Clarkson P, Abendstern M, Sutcliffe C, Hughes J, Challis D. The identification and detection of dementia and its correlates in a social services setting: Impact of a national policy in England. Dementia 2012; 11(5):617-32.

36. Chow TW, Binder C, Smyth S, Cohen S, Robillard A. 100 years after Alzheimer: Contemporary neurology practice assessment of referrals for dementia. Am J Alzheimers Dis Other Demen. 2009; 23(6): 516-27.

37. Boustani MC, Christopher U, Frederick A, Austrom MG, Perkins AJ, Fultz BA, et al. Implementing a screening and diagnosis program for dementia in primary care. J Gen Intern Med. 2005; 20(7): 572-7. 


\section{KOR PUI-KIN PATRICK ET AL.}

38. National Collaborating Centre for Mental Health (UK). Dementia: A NICE-SCIE Guideline on Supporting People With Dementia and Their Carers In Health And Social Care. Leicester (UK): British Psychological Society; 2007.

39. Nagaendran K, Christopher C, Chong MS, Chua EV, Goh CK, Kua J, et al. Ministry of health clinical practice guidelines: Dementia. Singapore Med J. 2013; 54(5): 293298; quiz 299. Erratum in: Singapore Med J. 2013; 54(8): 474.
* Corresponding author: Lai Kam-Yuk Claudia Tel: 852-2766 6544 Fax: 852-2364-9663

Email: claudia.lai@polyu.edu.hk. 